\title{
Normalización de la terminología mexicana sobre el agua
}

\author{
Catalina Naumis-Peña * \\ Verónica Vargas-Suárez **
}

Artículo recibido:

17 de mayo de 2009.

Artículo aceptado:

21 de octubre de 2009.

\section{RESUMEN}

Se expone el resultado de una investigación llevada a cabo para conocer el estado de la normalización terminológica en tesauros especializados que incluyen el agua dentro de sus áreas contratando los términos usados en la literatura mexicana. La documentación que se genera sobre el tema del agua en México crece en forma exponencial en tanto que la problemática del agua exige soluciones integrales. Actualmente no existe un tesauro, ni terminologías relacionadas con el tema del agua en México, ni tampoco una propuesta terminológica concreta sobre el tema en español. Por eso el presente documento aborda el tema del agua, la terminología

* Centro Universitario de Investigaciones Bibliotecológicas de la UNAM, México. naumis@servidor.unam.mx

** Instituto Mexicano de Tecnología del Agua, México. veronica_vargas@tlaloc.imta. $\mathrm{mx}$

INVESTIGACIÓN BIBLIOTECOLÓGICA, Vol. 23, Núm. 49, septiembre/diciembre, 2009, México, ISSN: 0187-358X. pp. 125-148 
que se usa en el español de México y los problemas que encuentra la intermediación terminológica que se realiza a partir de la Bibliotecología para representar estos contenidos en los sistemas de información.

Palabras clave: Normalización terminológica; Terminología sobre el agua.

\begin{abstract}
Standardization of Mexican terminology on water Catalina Naumis Peña and Verónica Vargas Suárez

The result of a research intended to know about the state of terminological standardization in specialized thesauri which include water within its areas, is exposed contrasting the terms used in Mexican literature of the field. Documentation generated over the theme of water in Mexico grows exponentially, but water problems keep demanding integral solutions. Presently neither a thesaurus nor a terminology over the theme of water exists in Mexico, nor a concrete terminological offer over this theme in Spanish. This paper confronts the water theme, the terminology used in the Mexican Spanish and the problems found in terminological intermediation based on Library Science in order to represent those contents in the information systems.
\end{abstract}

Keywords: Terminological standardization; Water terminology.

\title{
INTRODUCCIÓN
}

— 1 objetivo de esta investigación es la reutilización de recursos, ahí donde - 1 los esfuerzos invertidos en recopilar, organizar y sistematizar considerables volúmenes de información relacionada con el recurso hídrico no se limiten a la construcción de un instrumento aislado, sino que aporten nociones y denominaciones en la lengua especializada sobre el agua en México para generar o enriquecer otros recursos o desarrollar posteriormente un tesauro. ${ }^{1}$ 
Por su estructura, un tesauro es un vocabulario controlado y dinámico de términos relacionados semántica y genéricamente, los cuales cubren un dominio específico del conocimiento. La estructura de la terminología de un tesauro está basada en las interrelaciones existentes entre los conceptos. Con el control terminológico se busca neutralizar la sinonimia y la polisemia, características naturales de la lengua que dificultan la precisión de la indización y recuperación de la información, ambas funciones básicas de los tesauros.

La indización por asignación ${ }^{2}$ conlleva la utilización de un tesauro que, además de constituir la base del proceso para indizar y recuperar información, ayuda a fijar la terminología y la univocidad de cada uno de los términos que representan un concepto. La recuperación de los contenidos documentales puede hacerse por medio de los términos del lenguaje natural o por los términos generados en la indización por asignación. Es sabido que en esta segunda opción se cumplen los requisitos terminológicos que permiten satisfacer las necesidades de los usuarios con mayor precisión y menor pérdida de tiempo. Otro de los servicios que ofrece el tesauro es constituir la base terminológica y estructural para conformar las ontologías que establecerán las relaciones informáticas en los documentos digitales, como se puede observar en la ontología que se desarrolla para la base de datos del AGROVOC. ${ }^{3}$

Por otro lado un tesauro es una herramienta lingüística de alto costo por el tiempo y esfuerzo que supone la investigación y la construcción que lleva aparejada, y por el proceso de evaluación al que debe ser sometida una vez terminada. Tomando en cuenta lo anterior y la recomendación de los estudiosos se plantea la elaboración de un tesauro nuevo únicamente cuando se hayan agotado las posibilidades de adoptar y/o adaptar alguno ya existente.

Un tesauro es difícil de elaborar porque además de exigir un trabajo previo de diseño y mantenimiento, debe recopilar los términos usados para transferir conocimientos en la literatura de la disciplina y estudiarlos con la finalidad de definir los descriptores que reducen los contenidos documentales de manera tal, que un concepto esté siempre representado por el mismo término, manteniendo una red de relaciones entre descriptores y no-descriptores, que contextualicen los significados de cada uno de ellos. En el descriptor confluyen varios aspectos fundamentales: el contenido o representación del conocimiento (semántica); la definición del objeto que el descriptor designa (terminología y lexicografía); la estructuración dentro de una organización expresada a través de las relaciones conceptuales con otros descriptores 
(lingüística); la estructura morfo-sintáctica del descriptor (lingüística); la función de almacenar en un sistema de información (bibliotecológica); y la función de recuperador para satisfacer una necesidad de información del usuario (bibliotecológica). ${ }^{4}$

La experiencia en la indización de una colección de documentos sobre el tema del agua mostró un gran vacío en los lenguajes usados para representar este tipo de contenido temático, en lo fundamental porque algunos aspectos no estaban representados en ellos. En consecuencia ante el reto de organizar temáticamente una colección especializada en el tema del agua se analizaron descriptivamente seis tesauros relacionados con el tema hídrico, con el propósito de probar la necesidad de contar con una herramienta terminológica que representara la realidad actual mexicana.

El presente artículo se desarrolla en tres apartados en consecuencia con el objetivo anteriormente mencionado. En el primer apartado se presenta un esbozo de la problemática hídrica en México y la necesidad de organizar la información que se ha generado sobre el tema. En el segundo se analizan descriptivamente los seis tesauros seleccionados, así como 50 términos extraídos de 150 documentos relacionados con la temática hídrica. En el tercer apartado se discuten los resultados, y finalmente se presentan las conclusiones y perspectivas de investigación.

\section{LA INFORMACIÓN SOBRE EL TEMA DEL AGUA EN MÉXICO}

El recurso hídrico es escaso en muchas zonas del planeta, entre ellas México, y los conflictos sociales generados por su manejo crecen y se complican cada vez más. Esta situación se refleja en la terminología que representa no sólo los problemas del uso comunitario e individual del agua sino también los aspectos ambientales, sociales y económicos que se deben resolver.

Según la Comisión Nacional del Agua (Conagua) el crecimiento de la población y de las actividades económicas ha provocado una disminución sustancial en la disponibilidad per cápita de agua. La disponibilidad natural media per cápita, que resulta de dividir el valor nacional entre el número de habitantes, ha disminuido de $18035 \mathrm{~m}^{3}$ por habitante al año en 1950 a tan sólo 4312 en el 2007. La misma Conagua señala que a partir de la década de 1970 ha venido aumentando considerablemente el número de acuíferos sobreexplotados: de 32 en 1975, a 104 en 2006. Empero, en 2007 se redujo 
el número a 101. Cabe resaltar que de los acuíferos se extrae el 58\% del agua subterránea para todos los usos. ${ }^{6}$

Por otra parte, según datos de la Secretaría del Medio Ambiente y Recursos Naturales, la calidad del agua superficial, que es la única que se mide sistemáticamente, indica que $66 \%$ es excelente o aceptable mientras que el resto requiere tratamiento o se encuentra severamente contaminada. ${ }^{7}$

El tercer informe de las Naciones Unidas sobre el desarrollo de los recursos hídricos en el mundo, Water in a changing world (2009) coincide al señalar que, a nivel mundial, el problema de la disponibilidad efectiva del agua es aun mayor por los desequilibrios hidráulicos que ocasiona el constante crecimiento de la demanda, la ineficiencia de su uso y el aumento de los niveles de contaminación ocasionados por prácticas inadecuadas en los esquemas de producción y consumo. Además sostiene que debido al crecimiento de la población urbana, muchas grandes ciudades se han visto obligadas a importar agua de cuencas cada vez más lejanas, ya que las fuentes locales de aguas superficiales y subterráneas han dejado de satisfacer la demanda por agotamiento o contaminación.

Evidentemente es esencial sensibilizar a la sociedad ante esta situación. No hay que olvidar que la falta de una gestión sustentable del agua parte del desconocimiento de lo que se quiere proteger, cuidar y usar. Como lo advierte Corral Verdugo (citado por Vargas Pasaye),

La responsabilidad fundamental de la academia es la investigación y la difusión de los hallazgos científicos al respecto de las causas y las consecuencias de la crisis del agua. Les corresponde a los políticos, incluidos los universitarios (autoridades), hacer llegar a la sociedad la información y la tecnología (tanto física como social) derivada de este conocimiento. ${ }^{8}$

Indiscutiblemente una sociedad no informada es una sociedad no participativa, por lo que es trascendental que la información relacionada con el recurso hídrico esté a disposición de todos. Si se parte de la base de que la participación ciudadana es básica para solucionar la problemática hídrica, ${ }^{9}$ entonces es necesario que la información fluya hacia la sociedad con la finalidad

$6 \quad$ Ibid, p. 42.

7 B. Jiménez, et al, El agua en México vista desde la academia. México: Academia Mexicana de Ciencias, 2005, p.11.

8 Rafael G. Vargas Pasaye, (2005), “Los académicos alertan sobre el problema del agua”, en Campus suplemento universitario, México: UNAM, septiembre, Núm. 143. http://www.campusmilenio.com/, consulta: 04.09.05.

9 A este respecto, podemos mencionar las organizaciones barriales en Argentina, mencionadas por Olszewski et al. (2005), surgidas luego del crack institucional de fines de 2001. 
de que ésta tome conciencia sobre el valor ambiental, económico y social del recurso. Cierto es que el hecho de estar informado sobre la problemática real permite la sensibilización y crea el espacio y los elementos necesarios a través de los cuales los patrones de consumo, uso y conservación pueden modificarse.

Con el fin de aspirar a una gestión equitativa y sostenible del agua, tal como lo sugiere la UNESCO en los desafíos que presenta en su primer informe sobre el desarrollo de los recursos hídricos ${ }^{10}$ la investigación que se realiza en la actualidad en México aborda el tema del agua desde un enfoque holístico. Situación que obliga a contar con sistemas de información claros y consistentes que retroalimenten y reflejen el proceso que supone la transmisión de los resultados a la sociedad, pues la divulgación es una herramienta importante y necesaria.

La comunicación de los resultados pasa entonces por la organización de sistemas que los hagan de uso público. Se sabe que en los sistemas de información juega un papel central la recuperación temática de los contenidos de los documentos que exigen una indización a través de un medio que proporcione claridad y consistencia en las denominaciones de los temas de investigación. El resultado de contar con una herramienta lingüística que regule la terminología facilita el acceso útil a la información. Desde Wuster ${ }^{11}$ es conocido que los problemas de la representación no pueden ser resueltos con la simple traducción de tesauros que están en inglés. Asimismo el análisis de las nociones y de las denominaciones debe ser hecha por los diferentes discursos profesionales y académicos del español para acceder a la interpretación concreta de los diferentes términos de especialidad usados en los enunciados, porque se necesita la información contextual para obtener una interpretación similar a la que el documento pretende comunicar. ${ }^{12}$

\section{Metodología, ANÁLISIS Y RESUltAdos DE LA INVESTIGACióN}

A pesar de la aparición en escena de nuevos términos para designar los nuevos aspectos del tema, el enfoque social, político y económico se refleja muy poco en los tesauros de la especialidad y sobre todo en idioma español. Actualmente no existen herramientas lingüísticas sobre la temática hídrica que partan desde los diferentes enfoques disciplinarios. El resultado es que el

10 unESCO (2003), Agua para todos, agua para la vida.

11 Lerat Pierre, (1997), Las lenguas especializadas, Barcelona, Ariel p. 121.

12 Francisco Yus Ramos, (2007), "La comunicación en las lenguas de especialidad", p. 86 y Agustín Vera Luján, "El español profesional y académico”, p. 275, en Las lenguas profesionales y académicas. 
bibliotecólogo no cuenta con una herramienta que le permita tener mayor precisión y el rigor necesario para garantizar la calidad en los procesos de indizar y recuperar la información. Sin contar que existe una grave inconsistencia en los términos empleados en la literatura en castellano, lo que puede ocasionar una dispersión de documentos sobre un mismo tema.

Si bien el número de tesauros elegidos no pretende ser exhaustivo, dicha selección estuvo guiada por la relevancia de las herramientas y su utilización en entornos relacionados con el agua. Los tesauros seleccionados fueron: ${ }^{13}$

1. España, Ministerio de Fomento, Centro de Estudios y Experimentación de Obras Públicas, Tesauro de Ingeniería Hidráulica [en línea], Madrid: Cedex, 1998, [rev. 10 julio, 2009], disponible en: http://hispagua.cedex.es/

En este artículo referido como Hispagua.

2. European Environment Agency, General Multilingual Environmental Thesaurus [en línea], Copenhagen : EEA, 2004, [rev. 10 de julio, 2009], disponible en:

http://www.eionet.europa.eu/gemet .

En este artículo referido como GeMET.

3. FAO, Agrovoc Thesaurus [en línea], Rome: FAO, 198-, [rev. 9 de julio, 2009], disponible en:

$<$ http://www.fao.org/aims/ag_intro.htm>.

En este artículo referido como Agrovoc.

4. IRC International Water and Sanitation Centre. InterWATER Thesaurus, The Hague: IRC, 2006, [rev. 9 de julio, 2009], disponible en: $<$ http://www.thesaurus.watsan.net> .

En este artículo referido como InterWATER.

5. Organisation for Economic Co-operation and Development, OECD Macrothesaurus [en línea], París: OECD, 1991, [rev. 10 de julio, 2009], disponible en:

http://bibliotecavirtual.clacso.org.ar/ar/oecd-macroth .

En este artículo referido como OECD.

6. Organización Mundial de la Salud, Organización Panamericana de la Salud, Área de Desarrollo Sostenible y Salud Ambiental, Tesauro de Ingeniería Sanitaria y Ambiental, $17^{\mathrm{a}}$ ed. [Washington, DC : OPS], 298 p.

En este artículo referido como Repidisca.

13 Cabe señalar que el Water Resources Thesaurus, del Departamento del Interior de los Estados Unidos, no se incluyó, ya que su última edición es de 1980 y se encuentra fuera de circulación. Por su parte, el Thésaurus Eau, elaborado por la Oficina Internacional del Agua, no está disponible a través de la Internet. 
Dado que la gestión del agua debe tratarse desde un enfoque interdisciplinario, se ha tomado en cuenta en cada uno de los tesauros evaluados, como primera prioridad, la inclusión del tema del recurso hídrico desde todos los enfoques posibles. Los otros aspectos importantes a evaluar sobre cada una de las herramientas lingüísticas son: la institución que respalda al tesauro, tomando en cuenta su experiencia y el prestigio que goza dentro del medio científico y académico; y la disponibilidad y accesibilidad del tesauro, condiciones indispensables para poder llevar a cabo el estudio correspondiente. De igual manera, se consideraron tres factores principales:

1) el contenido, que alude a la cobertura temática, a su nivel de estructuración y a su mantenimiento y actualización;

2) la presentación, que se refiere a las características distintivas de cada tesauro y a la forma como éstas son tratadas, y

3) la consulta, la cual hace mención a la facilidad de manejo del tesauro.

El estudio fue enfocado mediante la consulta de cada uno de los tesauros, el análisis de su contenido, tomando en cuenta las necesidades de la organización documental sobre el agua en México, y el análisis descriptivo de los tesauros consultados en función del soporte que ofrecen para indizar los documentos.

Para realizar el análisis de contenido de los tesauros se compararon términos relacionados con el recurso hídrico seleccionados de aproximadamente 150 documentos generados en México y escogidos por la consulta frecuente de investigadores de diferentes ámbitos académicos, durante un periodo de observación de dos meses, sobre una colección especializada en el tema del agua $^{14}$ y de acuerdo con los lineamientos recomendados por Svenonius, ${ }^{15}$ quien hace notar que todo vocabulario controlado debe contar con una garantía literaria, (literary warrant), una garantía del usuario (user warrant o common usage) y una justificación de los expertos en el tema (scholarly usage).

De estos documentos se extrajeron términos que presentaban: la frecuencia de uso más alta en la colección, de acuerdo con su aparición reiterada en

14 A este respecto, Cabré menciona: "Una de las características más importantes de un texto especializado es la presencia de unidades terminológicas, cuanto más elevado sea el nivel de especialización de un texto, más alta será su densidad terminológica. Normalmente, un texto con un alto nivel de especialización es preciso, conciso y sistemático; la terminología que se utiliza en este texto tiene tendencia a la monosemia y a la univocidad. A medida que el grado de especialidad va disminuyendo, el discurso va adquiriendo características del discurso no especializado: desde el punto de vista semántico se observan variaciones conceptuales, redundancias, ambigüedades y una falta de precisión estricta; desde el punto de vista de la expresión, se observa un alto nivel de sinonimia, pero sobre todo un uso muy extendido de expresiones parafrásticas para expresar analíticamente un concepto que, en un nivel especializado, se podría expresar con un solo término, sin caer en la equivocación". 
los documentos, y los términos utilizados por los investigadores en las consultas documentales y en la discusión con ellos acerca de los términos escogidos para las estrategias de búsqueda. Después de conformar el listado de términos candidatos se buscaron dichas expresiones técnicas en los seis tesauros para analizar de qué manera eran tratados y estudiar las coincidencias en traducción y uso.

El estudio comenzó con un término no sólo de aparición frecuente en la literatura revisada, sino muy utilizada en México a nivel del discurso oral y que en cierto modo operó como disparador del proceso de investigación de la terminología sobre el tema, por lo que merece una mención especial. Dicho término es gestión integrada de los recursos hídricos, pues resume el tratamiento del tema del agua desde una perspectiva holística, considerando cada uno de sus aspectos, desde su aprovechamiento, uso y reuso, hasta los impactos ambientales, técnicos, políticos y sociales que ello conlleva para un ámbito geográfico específico.

Se trata de un término con un alto nivel de precoordinación para referirse a un gran problema que debe resolver la sociedad moderna y fue acuñado por el International Water Management Institute. En varios documentos escritos en español y analizados para el presente trabajo se observa la aparición de términos que pueden considerarse equivalentes y que son también empleados en los contenidos documentales: "manejo integrado de los recursos hídricos", "administración integrada de los recursos hídricos", "gestión integral de los recursos hídricos", "manejamiento integrado de los recursos hídricos", "gestión integral del recurso hídrico", "gestión integral del agua”, "gestión integrada del agua", "gestión integrada de recursos hidráulicos”, “ordenación integrada de los recursos hídricos", "gestionamiento integral de los recursos hídricos" y "gestión sostenible del agua".

En los seis tesauros consultados el término aparece solamente en uno como "gestión integrada de recursos hídricos", en otros dos se incluye como "gestión integrada" y en los tres restantes no fue incluido. Dentro de los tesauros multilingües estudiados, se observa que el término más empleado es Water management, que tiene diversas traducciones: "ordenación de aguas", "ordenamiento de las aguas", "gestión del agua”, "administración hídrica” y "gestión y administración del agua". Como se puede apreciar, el mismo término puede tener diferentes traducciones y connotaciones.

Es sabido que no es conveniente utilizar un tesauro para unos términos y otros para los demás porque se pierde consistencia y el control de términos equivalentes, lo que provoca la no existencia de univocidad. El hecho que el término haya sido localizado en uno de seis tesauros demuestra la poca consistencia que tiene. 
A continuación se presentan los 50 términos seleccionados como candidatos en los 150 artículos que representan los contenidos a indizar, pues se considera pertinente mostrar las diferencias que presentan los tesauros, ya que los candidatos a término demuestran la imposibilidad de elegir algún descriptor en particular para llevar a cabo la indización, y por ende la clasificación de la documentación sobre este recurso. Para representarlos en la tabla se dividieron los términos en cinco grupos con la finalidad de unificar los resultados en los tesauros investigados: 1) usados como descriptores; 2) no incluidos como descriptores; 3 ) incluidos con una diferencia en el uso de singular/plural (generalmente de plural a singular, aunque también en menor medida del plural al singular); 4) incluidos con palabras diferentes que no necesariamente dan una equivalencia semántica; y 5) incluidos como equivalentes no aceptados.

La simbología utilizada es la siguiente:

+ El término está incluido como descriptor en el tesauro.

- El término no está incluido en el tesauro.

\# El término está incluido como descriptor, pero difiere en el uso de singular/plural.

/ El término aparece expresado con otras palabras.

$=$ El término aparece como equivalente no aceptado como descriptor.

Cuadro ilustrativo: términos seleccionados como candidatos

\begin{tabular}{|c|l|c|c|c|c|c|c|}
\hline \multicolumn{2}{|c|}{ Términos candidatos } & Agrovoc & GeMET & Hispagua & InterWater & OECD & Repidisca \\
\hline 1 & Abastecimiento del agua & + & + & + & + & + & + \\
\hline 2 & Acuíferos & - & $\#$ & + & + & - & + \\
\hline 3 & Administración del agua & - & - & $/$ & $/$ & - & $/$ \\
\hline 4 & Aguas subterráneas & + & $\#$ & + & + & $\#$ & + \\
\hline 5 & Aguas superficiales & $\#$ & + & - & + & + & + \\
\hline 6 & Bancos de agua & - & - & - & $/$ & - & - \\
\hline 7 & Calidad del agua & + & + & + & + & + & + \\
\hline 8 & Calentamiento global & + & $/$ & - & - & - & $=$ \\
\hline 9 & Cambio climático & + & + & - & - & - & + \\
\hline 10 & Ciclo hidrológico & + & + & + & + & + & + \\
\hline 11 & Condiciones & - & - & - & - & - & - \\
\hline 12 & hidrometeorológicas & & & & & & \\
\hline 13 & Consejos de cuenca & $/$ & $/$ & - & - & - & - \\
\hline 14 & Conservación del agua & - & + & $/$ & + & + & + \\
\hline 15 & Contaminación del agua & + & + & + & + & + & + \\
\hline 16 & Cultura del agua & - & - & - & - & - & - \\
\hline 17 & Derechos de agua & $/$ & - & - & $/$ & $/$ & $/$ \\
\hline
\end{tabular}




\begin{tabular}{|c|c|c|c|c|c|c|c|}
\hline 18 & Desalinización & / & / & + & / & + & I \\
\hline 19 & Desarrollo sustentable & / & 1 & - & / & + & 1 \\
\hline 20 & Desastres naturales & + & + & - & - & + & + \\
\hline 21 & $\begin{array}{l}\text { Desastres provocados por } \\
\text { el hombre }\end{array}$ & - & + & - & - & I & + \\
\hline 22 & Desempeño ambiental & - & - & - & - & - & + \\
\hline 23 & Distritos de riego & - & - & - & - & - & - \\
\hline 24 & Economía del agua & - & - & + & - & - & - \\
\hline 25 & Educación ambiental & - & + & - & + & + & + \\
\hline 26 & Efectos del clima & - & + & - & - & I & + \\
\hline 27 & Embalses & + & + & + & - & + & + \\
\hline 28 & Evaluación ambiental & + & + & - & I & I & - \\
\hline 29 & $\begin{array}{l}\text { Eventos hidrometeorológi- } \\
\text { cos extremos }\end{array}$ & - & 1 & - & - & - & - \\
\hline 30 & Gestión ambiental & + & + & - & - & + & 1 \\
\hline 31 & Gestión de la escasez & - & - & - & - & - & - \\
\hline 32 & Gestión del agua & + & + & / & - & - & - \\
\hline 33 & Gestión del riesgo & - & - & - & - & - & - \\
\hline 34 & Gestión equitativa del agua & - & - & - & - & - & - \\
\hline 35 & $\begin{array}{l}\text { Gestión integrada de los } \\
\text { recursos hídricos }\end{array}$ & - & - & - & - & - & 1 \\
\hline 36 & Humedales & I & I & + & I & I & + \\
\hline 37 & Impacto ambiental & + & + & - & + & - & + \\
\hline 38 & Medición de caudales & - & - & - & I & - & + \\
\hline 39 & Mercados de agua & - & - & - & - & - & - \\
\hline 40 & Participación comunitaria & + & + & 1 & 1 & + & + \\
\hline 41 & Presas & + & + & l & 1 & + & + \\
\hline 42 & Privatización del agua & I & I & - & 1 & I & I \\
\hline 43 & Reciclaje del agua & I & l & - & + & l & + \\
\hline 44 & Riego & + & + & $\#$ & + & + & + \\
\hline 45 & $\begin{array}{l}\text { Riesgos hidrometeoroló- } \\
\text { gicos }\end{array}$ & I & - & - & - & - & - \\
\hline 46 & Sequía & + & $\#$ & - & $\#$ & + & $\#$ \\
\hline 47 & $\begin{array}{l}\text { Tratamiento de aguas resi- } \\
\text { duales }\end{array}$ & + & + & I & + & - & + \\
\hline 48 & Tratamiento del agua & I & + & $\#$ & + & + & + \\
\hline 49 & Turismo ecológico & I & + & - & - & - & - \\
\hline 50 & Uso eficiente del agua & - & - & - & - & - & + \\
\hline
\end{tabular}

Fig. 1. Términos candidatos confrontados en los seis tesauros 


\begin{tabular}{|c|c|c|c|c|c|c|}
\cline { 2 - 7 } \multicolumn{1}{c|}{} & $\begin{array}{c}\text { Agrovoc } \\
\%\end{array}$ & $\begin{array}{c}\text { GeMET } \\
\%\end{array}$ & $\begin{array}{c}\text { Hispagua } \\
\%\end{array}$ & $\begin{array}{c}\text { InterWater } \\
\%\end{array}$ & $\begin{array}{c}\text { 0ECD } \\
\%\end{array}$ & $\begin{array}{c}\text { Repidisca } \\
\%\end{array}$ \\
\hline+ & 36 & 44 & 20 & 28 & 34 & 50 \\
\hline- & 42 & 34 & 64 & 48 & 50 & 32 \\
\hline$\#$ & 2 & 6 & 4 & 2 & 2 & 2 \\
\hline$/$ & 20 & 16 & 12 & 22 & 14 & 14 \\
\hline$=$ & 0 & 0 & 0 & 0 & 0 & 2 \\
\hline
\end{tabular}

Fig. 2. Porcentaje de incidencia según los cinco grupos establecidos

Agrovoc

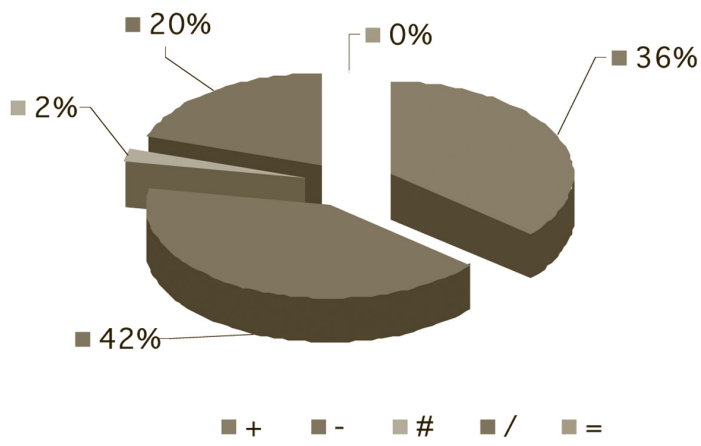

Fig. 3. Porcentaje de incidencia de los términos candidatos en el Agrovoc

Se observa que en el tesauro de Agrovoc el $42 \%$ de los términos candidatos no aparecen como descriptores. El 36\% de estos términos está incluido como descriptor en la herramienta; el 20\% de ellos aparece designado como concepto, con diferente expresión terminológica, el 2\% aparece como descriptor pero con diferencias entre singular y plural y no aparecen términos aceptados como equivalentes.

\section{GeMET}

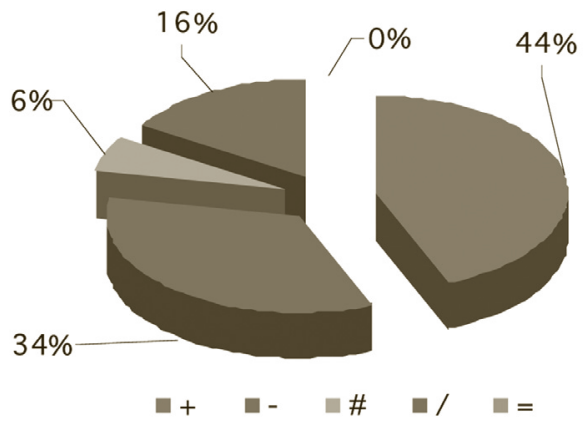

Fig. 4. Porcentaje de incidencia de los términos candidatos en el GeMET 
Se observa que en el tesauro de GeMET el 44\% de los términos candidatos aparecen como descriptores. El 34\% de estos términos no están incluidos como descriptores en la herramienta; el 16\% de los mismos aparecen designados como conceptos, con diferente expresión terminológica; el 6\% aparecen como descriptores pero con diferencias entre singular y plural, y no aparecen términos aceptados como equivalentes.

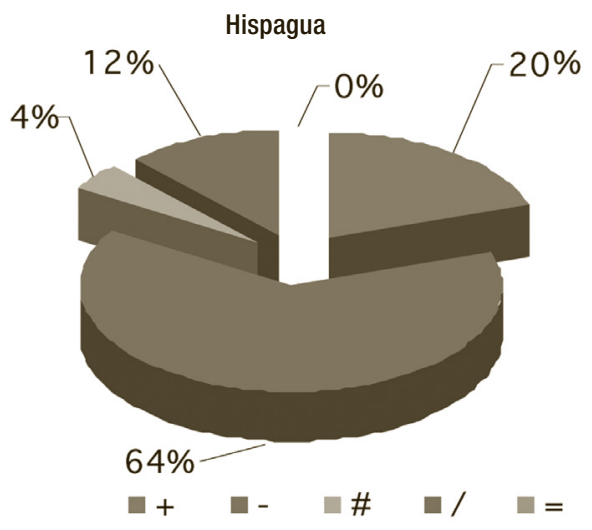

Fig. 5. Porcentaje de incidencia de los términos candidatos en el Hispagua

En el tesauro de Hispagua el 64\% de los términos candidatos no aparecen como descriptores; el 20\% de estos términos están incluidos como descriptores en la herramienta; el 12\% de los mismos aparecen designados como conceptos pero con diferente expresión terminológica; el 4\% aparecen como descriptores pero con diferencias entre singular y plural, y no aparecen términos aceptados como equivalentes.

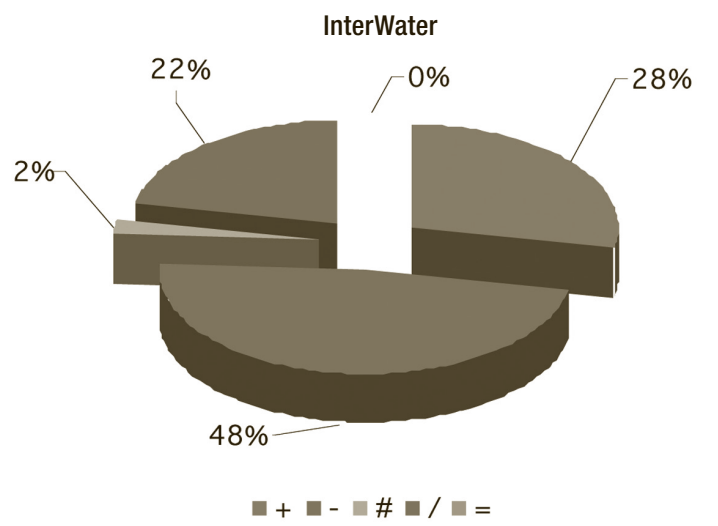

Fig. 6. Porcentaje de incidencia de los términos candidatos en el InterWater 
En el tesauro de InterWater el $48 \%$ de los términos candidatos no aparecen como descriptores; el $28 \%$ de estos términos están incluidos como descriptores en la herramienta; el 22\% de los mismos aparecen designados como conceptos, con diferente expresión terminológica; el 2\% aparecen como descriptores pero con diferencias entre singular y plural, y no aparecen términos aceptados como equivalentes.

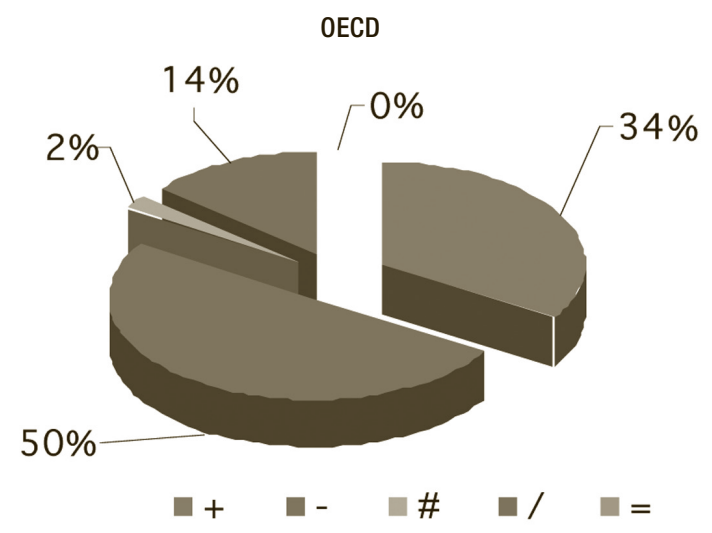

Fig. 7. Porcentaje de incidencia de los términos candidatos en el OECD

En el tesauro de la OECD el 50\% de los términos candidatos no aparecen como descriptores; el 34\% de ellos están incluidos como descriptores en la herramienta; el 14\% de los mismos aparecen designados como conceptos, con diferente expresión terminológica; el 2\% aparecen como descriptores pero con diferencias entre singular y plural, y no aparecen términos aceptados como equivalentes.

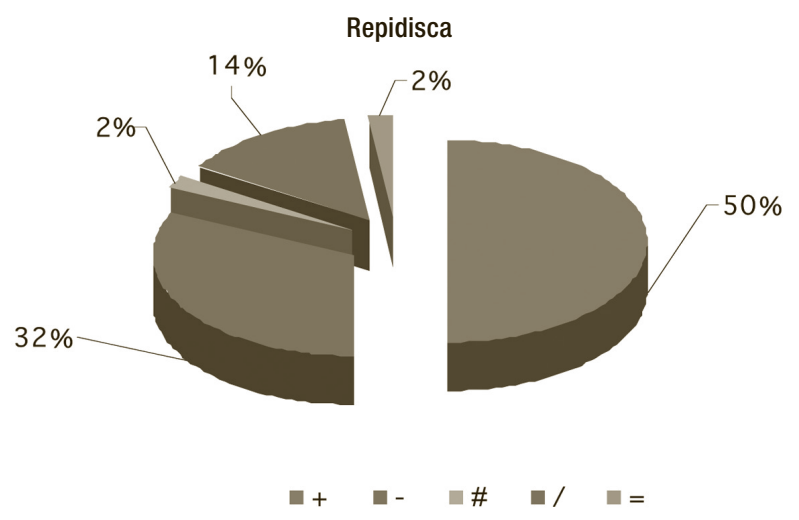

Fig. 8. Porcentaje de incidencia de los términos candidatos en el Repidisca 
Y en el tesauro de Repidisca el 50\% de los términos candidatos aparecen como descriptores; el 32\% de ellos no están incluidos como descriptores en la herramienta; el 14\% de los mismos aparecen designados como conceptos, con diferente expresión terminológica; el 2\% aparecen como descriptores pero con diferencias entre singular y plural, y un $2 \%$ aparecen términos de los candidatos en México aceptados como equivalentes.

\section{DisCusión DE LOS RESULTADOS}

Como se mencionó anteriormente un tesauro debe servir para tener una referencia rápida y confiable en un vocabulario predefinido que facilite la localización y la recuperación de información, para guiar la búsqueda de un tema específico usando las relaciones de significado entre los términos con la finalidad de clarificar los conceptos y temas relacionados con una materia en particular. Las características inherentes a un instrumento como el tesauro, que ofrece una información estructurada de forma consistente, permiten encontrar todos los posibles documentos relacionados con un concepto dado, porque siempre que cada uno de estos documentos esté asociado con un término o vinculado con un grupo de términos sinónimos el tesauro debe indicar estas relaciones.

En el análisis llevado a cabo resulta llamativo que sólo un tesauro cubra la mitad de los términos seleccionados. Los demás están por debajo del 50 por ciento. De igual manera, cabe mencionar que sólo en dos tesauros el porcentaje de términos incluidos es mayor al porcentaje de términos que no han sido tomados en cuenta, ni siquiera como términos no autorizados o no-descriptores. Al respecto, se ha de recordar que no es conveniente emplear un tesauro para unos términos y otros para los demás, porque se pierde tanto la consistencia como el control de términos equivalentes que tienen como consecuencia a la falta de univocidad.

En los términos candidatos analizados saltan a la vista las diferencias en el uso de plurales y singulares. Es evidente la falta de consenso en la aplicación de las normas ISO 2788 en la construcción de tesauros porque éstas indican que deben ir en plural los objetos contables y los grupos; y en singular los nombres abstractos y los fenómenos, así como las disciplinas, las ideologías y las creencias, los procesos y las actividades, y los objetos concretos no contables. Como ejemplo se pueden citar los términos "aguas superficiales" y "aguas subterráneas", el primero de los cuales en algunos tesauros se emplea en singular, y el segundo en plural o viceversa.

También se observan en el presente estudio las diferencias en la traducción. Baste mencionar que la mayoría de los tesauros consultados fueron elaborados 
originalmente en inglés y, que al estar realizando este análisis, en varias ocasiones se tuvo que buscar el término en esa lengua para poder localizar el término en castellano, pues la traducción no coincidía con el vocablo empleado en México.

El estudio comprendió además el análisis descriptivo de los tesauros para observar aspectos tales como las decisiones y políticas adoptadas en la construcción de ellos, y la explicación del servicio que proporciona el tesauro en la introducción, el mantenimiento, la actualización, la convocatoria al usuario y la traducción.

Al revisar la introducción, se advirtió que algunos de los tesauros no contaban con ella y que la falta de la misma, ${ }^{16}$ así como de la guía de empleo, dificultaba y limitaba el uso del tesauro. Es difícil detectar detalles de la construcción del tesauro aplicando únicamente los términos de la indización; es necesario contar con datos previos a la decisión de adoptarlo, como son las categorías principales, la cantidad de términos incluidos en cada una de éstas, las reglas para establecer relaciones entre términos, la cantidad de equivalencias, las políticas de inclusión, las instrucciones a los usuarios y la invitación para enviar propuestas de términos candidatos.

La importancia de tomar en cuenta la evolución del lenguaje científico y técnico es fundamental para indizar y esto queda reflejado en el interés demostrado por los equipos de construcción del tesauro por lo que se refiere a la evaluación, el mantenimiento y la actualización del mismo. Vale mencionar que la actualización del tesauro debe hacerse tanto para incorporar la terminología derivada del desarrollo de la ciencia o disciplina a la que se dedica, como para cubrir lagunas o fallas detectadas durante su utilización, y que hay que adaptarlo a las necesidades de recuperación manifestadas por los usuarios a través de sus búsquedas.

También se advierte que la convocatoria para proponer nuevos términos, que surge desde los tesauros hacia sus usuarios, demuestra la importancia de que el usuario se reconozca en sus necesidades de información. No hay que olvidar que el diseño de un lenguaje documental que no tenga en cuenta el contexto del usuario estará incompleto y dará origen a un lenguaje deficiente en sus prestaciones a los sistemas de recuperación de información.

Lo mismo sucede con el uso de extranjerismos o traducciones deficientes, pues sin temor a exagerar se puede afirmar que esto puede provocar confusiones

16 Al respecto Foskett (1997: 133) menciona que "The thesaurus can take several different forms. In every case, it will require an introductory section which explains how it can best be used. If this is not provided, the unlucky users will have to produce one for themselves. An introduction should therefore aim to cover all the sections: the limits of the field or fields covered, the choice and forms of terms, the network of relations, the form of presentation, and the use of special features like the graphical displays". 
en el empleo de los términos, lo cual sin duda empobrecería la calidad del tesauro y debilitaría su credibilidad, si tomamos en cuenta que un tesauro también puede ser utilizado como una herramienta de traducción, como lo menciona Domènec Turuguet (1994)

Las traducciones crean problemas adicionales: a menudo un término del tesauro original da lugar a dos o más traducidos, es difícil tener equivalencias perfectas y quizá algunos términos sean intraducibles. Como solución provisional puede usarse el tesauro original, especialmente si se trata de materias científicas y técnicas, pero en temas humanísticos la dificultad resulta notoria. Sin embargo, la existencia de un tesauro temáticamente afín al que se pretende elaborar siempre es una buena ayuda.

Una expresión representativa de los problemas de traducción es "humedales" (wetlands), que ha sido traducido también como "tierras húmedas", "ciénagas", "pantanos" y "tierras pantanosas"; términos que no necesariamente tienen el mismo significado de lo que se entiende por humedal, según la Convención de Ramsar. ${ }^{17}$

Esto también sucede con expresiones como "calentamiento global" y "efecto invernadero" que se emplean como si fueran sinónimos, cuando cada una tiene su propio significado. Lo mismo sucede con "desarrollo sostenible" y "desarrollo sustentable". A este respecto puede tomarse en cuenta lo que menciona Ramón Margalef, ${ }^{18}$ que aun sin quererlo, la repetición de un término lleva cada vez a diferentes connotaciones.

Otro aspecto que ha llamado nuestra atención es la omisión de términos relacionados con las ciencias sociales. Es decir, en la mayoría de los tesauros el recurso hídrico se contempla básicamente desde el punto de vista de las ciencias exactas, y se le da poca importancia a los aspectos sociales y económicos. Ejemplos de ello son la exclusión de vocablos como "conflictos por agua", "consejos de cuenca", "distritos de riego", "cultura del agua” y "gestión equitativa del agua”, entre otros.

Cabe mencionar que dichos conceptos se encuentran cada vez con mayor frecuencia en la literatura mexicana, más aún ante la creciente tendencia de atribuirles a las inadecuadas políticas públicas la responsabilidad de los problemas relacionados con el agua, lo que subraya la necesidad de insistir en la

17 De acuerdo con la Convención de Importancia Internacional sobre los Humedales, llamada la Convención de Ramsar, "las extensiones de marismas, pantanos y turberas, o superficies cubiertas de aguas, sean éstas de régimen natural o artificial, permanentes o temporales, estancadas o corrientes, dulces, salobres o saladas, incluidas las extensiones de agua marina cuya profundidad en marea baja no exceda de seis metros" son humedales.

18 Ramón Margalef i López, Teoría de los sistemas ecológicos, 1993, p. 254. 
importancia del comportamiento humano como un componente más de los sistemas de los recursos hídricos. El agua dulce es tan esencial para el desarrollo duradero como lo es para la vida y aparte de sus funciones geofísica, química y biológica en el ciclo hidrológico, tiene valores sociales, económicos y ecológicos interrelacionados que se respaldan mutuamente.

Durante el análisis se observó un incremento significativo de no-descriptores de una edición a otra, lo que refleja la necesidad de aproximar el lenguaje natural al lenguaje controlado. Una razón de esto podría ser que en la actualidad la mayoría de los usuarios realizan sus búsquedas de información directamente en las bases de datos, y de este modo el manejo de las equivalencias lingüísticas enriquece las posibilidades de recuperar contenidos relevantes. Otro aspecto importante es el surgimiento de modismos que en ocasiones están estrechamente relacionados con el cambio del discurso político. Cuando un término tiene cierto desprestigio o poco impacto en los gobernados, sus gobernantes escogen otro para sustituirlo.

De igual modo, como resultado del estudio se observaron además diferencias idiomáticas que reflejaban el contexto geográfico, social y el discurso político acerca del aprovechamiento y la existencia del agua como un recurso fundamental, trascendente y prioritario de la vida del ser humano en el planeta.

Esta investigación permitió determinar que el recurso hídrico en los tesauros existentes se contempla básicamente desde el punto de vista de las ciencias exactas, y que se le da poca importancia a los aspectos sociales, políticos y económicos que sin embargo juegan un papel fundamental en la actualidad. Al respecto cabe señalar que aunque las soluciones a los problemas del agua deben incluir temas tecnológicos es claro que el del agua, al ser un problema con un origen y una solución sociales, sólo podrá enfrentarse de manera efectiva empleando también la ingeniería social, desde el punto de vista de las ciencias políticas.

Por último se debe hacer notar que la creciente consolidación y expansión del entorno digital evidencia cada vez más la necesidad de tener una mayor disponibilidad y accesibilidad a los tesauros en línea, por lo que actualmente es difícil concebir un tesauro sólo en versión impresa. Los usuarios requieren de herramientas conceptuales y semánticas destinadas a una efectiva organización de la información, y los tesauros son una de las estructuras que pueden tener una participación activa en este sentido. Sin olvidar la usabilidad, utilidad y operabilidad en el diseño del tesauro, así como su evaluación y mantenimiento, cabe recordar que una forma de propiciar el uso de la información en la sociedad es favorecer su disponibilidad y fácil acceso.

Desde esta perspectiva, los aportes del entorno digital a los tesauros son considerables, como lo es el enriquecimiento de la funcionalidad de la estructura de 
los tesauros a partir de la hipertextualidad, además de la reducción de costos en cuanto al mantenimiento y la actualización. De igual manera la integración del usuario al proceso de creación, evaluación y optimización de los tesauros a través de pruebas de utilidad, uso de técnicas centradas en el usuario, etc., permite elaborar herramientas que tengan en cuenta los requerimientos de los usuarios y descartar su elaboración como simples estructuras teóricas.

\section{Consideraciones finales}

De los resultados obtenidos se puede inferir que no es factible adaptar algún tesauro en particular, no obstante resulta posible tomar lo más relevante de todos ellos y adaptarlo a la realidad mexicana, pues si bien es cierto que hay tesauros enfocados de manera general al agua, se debe tener presente que éstos han sido concebidos con el fin de satisfacer necesidades regionales y por tanto una parte de su terminología queda necesariamente lejos de la realidad de México. Esto equivale a decir que actualmente no existe una herramienta en español que refleje la terminología que se presenta en la investigación mexicana, pues los tesauros que se han elaborado en otros idiomas reflejan los enfoques geográficos y sociales de aquellas culturas que los han generado.

En la concepción de la terminología para transferir los contenidos documentales se observa un choque entre la Bibliotecología y la Terminología, ya que aunque la Bibliotecología sí puede proponer sistemas universales, está supeditada a los contenidos particulares que debe representar, a través de descriptores unívocos y sólo referidos a la variedad del lenguaje tanto en el mismo sistema de información como en otros contextos. Este conflicto fue analizado desde el punto de vista de la Terminología por Cardero. ${ }^{19}$ Por lo pronto la Bibliotecología debe rescatar los términos de la ciencia y la tecnología a nivel universal, pero de esa masa separar, al mismo tiempo, los términos particulares de un sistema de información que tengan características locales. ${ }^{20}$.

La Terminología, al estudiar la comunicación técnica a través de sus términos, acepta todas las variantes lingüísticas, tal como lo menciona Cabré:

El conocimiento científico, considerado como universalmente homogéneo, es el modelo que se debe seguir para organizar los conceptos de todas las materias profesionales, sin considerar las diferencias que presentan las distintas materias, contextos

19 Ana María Cardero, (2009), "El descriptor y el término: los conceptos y la lingüística".

20 Agustín Vera Luján, "El español profesional y académico", p. 275, en Las lenguas profesionales y académicas. 
socioculturales, áreas geográficas, realidades socioeconómicas y lenguas, tanto por su tipología como por su estatus social.

Uno de los problemas observados en los tesauros analizados, elaborados en un idioma diferente al español y con traducciones al mismo, es que las equivalencias lingüísticas no siempre son consistentes y reflejan el uso sólo en algunos de los países de habla hispana, por lo que no ofrecen una traducción válida para todos y cada uno de éstos países.

El estudio también ha mostrado la carencia de un vocabulario controlado en español sobre la temática hídrica que aborde el asunto de una manera holística, aunque tampoco se ha reflejado en los tesauros este enfoque. Esto ha provocado que el analista de información no cuente con una herramienta que le permita una mayor precisión y el rigor necesario para garantizar la calidad de los procesos de indización y recuperación de la información en el tema del agua.

Es decir, si bien existe una grave inconsistencia en los términos utilizados en la literatura en castellano, ésta se multiplica cuando se quieren utilizar términos traducidos de tesauros escritos en otros idiomas, pues, como es sabido, las inconsistencias en la terminología no permiten organizar los documentos generados en el tema de forma coherente y comprensiva para el medio cultural en el cual se producen. La terminología usada en los resultados de la investigación y observación de los problemas del agua en México muestra características específicas que crean la necesidad de contar con una herramienta terminológica pertinente.

Esta investigación también permitió observar que el tesauro admite una mayor especificidad de los términos relativos a campos disciplinares determinados, lo que implica un análisis más preciso de los documentos. De igual modo hizo posible incorporar las relaciones asociativas que favorecen la versatilidad y ayudan a crear un universo real e interdisciplinar como es el lenguaje científico. Es decir, la sistematización y transferencia del conocimiento, la traducción de textos científicos, y la formulación, diseminación, almacenamiento y recuperación de la información técnico-científica son actividades que se apoyan básicamente en el trabajo terminológico. Y es precisamente en relación con este último aspecto que surge el desarrollo de tesauros, los cuales están constituidos por términos que obedecen a reglas terminológicas propias, unidos entre sí por relaciones semánticas.

El tema a indizar y recuperar en los sistemas de información estudiado en este trabajo, además de ser un recurso vital para todo ser vivo, es indispensable para el desarrollo social y económico de cualquier comunidad. El problema de la escasez del recurso hídrico en México, y a nivel mundial, se percibe 
cada vez más grave y es más común y cercano. Actualmente México enfrenta problemas tanto de escasez como de contaminación de sus recursos hídricos, razón por la cual es imperioso difundirlos ante el usuario final a través de la disponibilidad de información organizada, para que cuente con una búsqueda generalizada de soluciones.

De igual manera la presión cada vez mayor a la que está sometido en México y en el mundo el recurso hídrico, en una situación de cambios antropogénicos y climáticos, exige enfocar de manera multidisciplinaria, integrada y dinámica los problemas científicos y sociales que plantea la problemática del agua, como lo demuestra la literatura que se ha venido generando en los últimos tiempos y que se ha revisado para esta investigación.

En síntesis, la problemática del agua en México, así como la falta de uniformidad en los términos empleados y la inexistencia de un vocabulario controlado en el español mexicano sobre el tema, limita su difusión a través de la indización de los contenidos documentales en forma consistente y clara en los sistemas de información, y en consecuencia afecta al proceso de socialización del conocimiento.

Entre los beneficios que brindaría el empleo de un tesauro del agua, creado expresamente para México, estaría la optimización de las labores de indización que se realizan en los centros de información y documentación relacionados con la temática hídrica. Hasta ahora estas labores siguen, por una parte, los esquemas de clasificación establecidos por instrumentos bibliotecológicos de índole general, no adaptados a un lenguaje especializado; y por la otra, en su mayoría toman como base para traducir los términos, aquellos utilizados en el lenguaje natural empleado por el autor. Esto ocasiona el uso de un mismo término para diferentes conceptos, o de muchos términos para un solo concepto, lo cual, a su vez, genera imprecisión. Y ésta es justamente una característica que no debe poseer un término técnico debido a que el usuario que lo utiliza, por ser especialista en un área determinada, requiere exactitud en su definición.

Tomando en cuenta que por su propia naturaleza el tesauro es una herramienta dinámica, observamos que en un contexto automatizado el uso de un vocabulario controlado resulta adecuado debido a la literalidad con la que los programas informáticos almacenan, organizan y recuperan la información, y porque el tesauro permite combinar los términos en la recuperación. Además, se comprueba que en una sociedad globalizada, donde la comunicación se manifiesta cada vez más de manera intercultural, el rescate y análisis del lenguaje técnico se convierte en una herramienta fundamental para servir de base en la elaboración de tesauros adaptados a las necesidades de indización y recuperación de información en contextos particulares. 
A través de esta investigación se ha podido observar que la construcción de tesauros, a pesar de ser una ardua labor, produce frutos invaluables. En tal sentido, un tesauro relativo a la temática hídrica, tratada de manera holística, puede sentar las bases para crear una terminología propia y, sin temor a exagerar, contribuir al desarrollo del idioma español.

En este análisis sólo hemos ejemplificado una porción de los tesauros que pueden servir de base para formalizar la elaboración de un tesauro del agua para México, pero el aporte más significativo del mismo son los términos frecuentes en uso en el lenguaje especializado sobre el tema en el contexto mexicano.

\section{REFERENCIAS BIBLIOGRÁFICAS}

Aitchison, J., A. Gilchrist and D. Bowden (2000), Thesaurus construction and use: A practical manual, Chicago: Fitzroy Dearbon.

Cabré, María Teresa (2002), "Terminología y lingüística: la teoría de las puertas”, en Estudios de lingüística española, volumen 16.

Cardero, Ana María (2009), "El descriptor y el término. Los conceptos y la lingüística”, pp. 53-62, en Memorias del I Simposio Internacional sobre Organización del conocimiento: Bibliotecología y Terminología, Introducción y coordinación de Catalina Naumis Peña, México: Centro Universitario de Investigaciones Bibliotecológicas.

Comisión Nacional del Agua (2008), Estadísticas del agua en México 2008.

España, Ministerio de Fomento, Centro de Estudios y Experimentación de Obras Públicas, Tesauro de Ingeniería Hidráulica [en línea], Madrid : Cedex, 1998, [rev. 10 julio, 2009], disponible en: http://hispagua.cedex.es/ .

European Environment Agency, General Multilingual Environmental Thesaurus, Copenhagen: EEA, 2004, [rev. 10 de julio, 2009], disponible en: <http://www.eionet.europa.eu/gemet> .

FAO, Agrovoc Thesaurus, Roma : FAO, 1980, [rev. 9 de julio, 2009], disponible en: <http://www.fao.org/aims/ag_intro.htm>.

Foskett, D.J. (1997), Thesaurus, en Readings in information retrieval, ed. K. Sparck Jones and P. Willet, San Francisco: Morgan Kaufmann, pp.111-34.

Hjørland, Birger (2002), "Domain analysis in information science: Eleven approaches - traditional as well as innovative", en Journal of Documentation, 58(4):422-62.

International Organization for Standardization (1986), ISO 27881986(E) Documentation - Guidelines for the establishment and development of monolingual thesauri, 2nd ed., Geneva: ISO, 1986, 32 p. 
IRC International Water and Sanitation Centre, InterWATER Thesaurus, The Hague : IRC, 2006, [rev. 9 de julio, 2009], disponible en: $<$ http://www.thesaurus.watsan.net> .

Jiménez, Blanca et al, (2005), El agua en México vista desde la academia, México: Academia Mexicana de Ciencias, 2005.

Jorna, K. and S. Davies (2001), "Multilingual thesauri for the modern world - no ideal solution?", en Journal of Documentation 57(2): 284-95.

Lancaster, F.W. (2001), "Indización de documentos científicos", en Procesamiento de la información cientifica, Madrid: Arco Libros, pp. 164-181.

Lerat, Pierre (1997), Las lenguas especializadas, trad. Albert Ribas, Barcelona: Ariel. 221 p.

16) López-Huertas, M. J. (1997), "Thesaurus structure design: A conceptual approach for improved interaction", en Journal of Documentation 53(2): 139-77.

Margalef I López, Ramón (1993), Teoría de los sistemas ecológicos.

Naumis, C. (2002), Modelo de construcción de tesauros documentales multimedia: aplicaciones a los contenidos educativos en televisión, Madrid: Universidad Complutense de Madrid.

Naumis, C. (2007), Los tesauros documentales: y su aplicación en la información impresa, digital y multimedia, Buenos Aires: Alfagrama.

National Information Standards Organization (2003), Guidelines for the Construction, Format, and Management of Monolingual Thesauri, Bethesda: NISO.

Olszewski, Ana et al. (2005), Agua para todos: gestión participativa como expresión de intereses y necesidades sociales, Buenos Aires: Espacio Editorial.

Organización Mundial de la Salud, Organización Panamericana de la Salud. Área de Desarrollo Sostenible y Salud Ambiental, Tesauro de Ingeniería Sanitaria y Ambiental. $17^{\mathrm{a}}$ ed. [Washington, DC: OPS], $298 \mathrm{p}$.

Organización para la Cooperación y el Desarrollo Económico, OECD Macrothesaurus, Washington, DC: OECD, 1991. [rev. 10 de julio, 2009], disponible en: <http://bibliotecavirtual.clacso.org.ar/ar/ oecd-macroth> .

Owens, L. A., and P. A. Cochrane (2004), “Thesaurus evaluation”, en Cataloging E Classification Quarterly, 37(3/4):87-102.

Shiri, Ali Ashgar; Crawford Revie (2000), "Thesauri on the Web: current developments and trends", en Online Information Review, 24(4): 273-279.

Svenonius, E. (2003), "Design of controlled vocabularies", en Encyclopedia of Library and Information Science, New York: Marcel Dekker, pp.822-838. 
Turuguet, Domènec (1994), "Consideraciones sobre los tesauros”, en El profesional de la información, n. 21, [rev. 30 de abril, 2009], disponible en línea: http://www.elprofesionaldelainformacion.com/ contenidos/1994/febrero/consideraciones_sobre_los_tesauros. html.

unESCO, Programa Mundial de Evaluación de los Recursos Hídri$\cos (2003)$, Agua para todos, agua para la vida: informe de las Naciones Unidas sobre el desarrollo de los recursos hidricos en el mundo, París: UNESCO.

UnesCO, World Water Assessment Programme (2009), The United Nations World Water Development Report 3: Water in a Changing World, [rev. 3 de agosto, 2009], disponible en: http://www.unesco.org/water/wwap/wwdr/ .

Vargas Pasaye, Rafael G. (2005), "Los académicos alertan sobre el problema del agua", en Campus suplemento universitario, México: UNAM, septiembre, núm. 143 [rev. 4 de septiembre, 2005], disponible en: http://www.campusmilenio.com/ .

Vargas Suárez, Verónica (2007), Análisis del lenguaje relacionado con el agua: necesidad de un tesauro para México, México: Universidad Nacional Autónoma de México.

Vera Luján, Agustín (2007), "El español profesional y académico", en Alcaráz Varó, Enrique, Mateo Martínez, José y Francisco Yus Ramos, Las lenguas profesionales, pp. 271-279.

Yus Ramos, Francisco (2007), "La comunicación en las lenguas de especialidad”, en Alcaráz Varó, Enrique, Mateo Martínez, José y Francisco Yus Ramos, Las lenguas profesionales, Barcelona: IULMA. pp. 81- 92. 